\title{
Circuit models of Lossy Coaxial Shielded cables to Analyze Radiated and Conducted Susceptibilities with unmatched line loads
}

\author{
M.SAIH ${ }^{1}$, H.ROUIJAA ${ }^{2}$, A.GHAMMAZ ${ }^{1}$, \\ ${ }^{1}$ Laboratory of Electrical Systems and Telecommunications, Department of Physics, Faculty of \\ Sciences and Technology, Cadi Ayyad university, Marrakesh, Morocco \\ ${ }^{2}$ Laboratory of Electrical Systems and Telecommunications, Department of Applied Physics, Faculty \\ of Sciences and Technology, Hassan ler university, Settat, Morocco
}

\begin{abstract}
This paper presents a circuit models to analyze the variation effects of incident plane wave on shielded coaxial cables, using Branin's method, which is called the method of characteristics. The model can be directly used for the time-domain and frequency-domain analyses and for all arbitrarily loaded. This makes it easy to insert in circuit simulators, such as SPICE, SABER, and ESACAP. The obtained results are in good agreement with those from others methods. Finally, we will discuss the effects of the variation of the incident plane wave.
\end{abstract}

Index Terms - Plane wave, shielded coaxial cables, method of characteristics, transfer impedance, Circuit models.

\section{INTRODUCTION}

Shielded coaxial shielded cables are usually used in RF and microwave circuits as resonators [1], VLSI interconnect [2], wave shaping [3] analog signal processing [4],filters [5] and etc. Nevertheless, there exists coupling between the exterior and interior of the shield for the reason that the imperfect nature of the shield. Consequently, electromagnetic interference (EMI) and electromagnetic compatibility (EMC) problems associated with cables [6] connecting these devices should be taken into consideration.

Circuit models for multiconductor transmission lines (MTLs) with and without shields [7], [8] have been a subject of great interest in recent years. Spice models to analyze the conducted immunity of coaxial cables have been presented in [9], then some spice models have presented for the analyses of the conducted and radiated immunity of lossless shielded cables [10]. These models are not inherently capable to analyze directly the time-domain, the inverse Fourier transform (IFT) is needed for the models to obtain the time-domain results. Recently, some Spice models have been proposed to analyze the conducted and the radiated susceptibilities of lossless shielded coaxial cables [8], [11].The principle point of interest of these models comprises in the likelihood of utilizing them as a part of frequency and time domains, with linear and non linear loads individually, and the discretization of shielded cables is not needed. However, these models cannot be used to analyze lossy shielded cables. After Then, some lossy models have been presented to analyze the conducted and radiated 
susceptibilities of Multiconductor Shielded cables [12]. However, a similarity transformation is needed to decouple the inner transmission-line equations.

In this paper, an equivalent circuit models to analyze the radiated susceptibility of uniform shielded coaxial shielded cables is presented. These models can be used to analyze both the time-domain and frequency domain and for all arbitrarily loaded. There is a good correlation with those from other methods.

\section{DESCRIPTION OF COAXIAL SHIELDED CABLES}

\section{A. Model of shielded cables}

For a coaxial cable over an infinite and perfectly conducting, as illustrated in Fig. 1, the coupling with external fields can be described by [8]:

Outer system

$$
\left\{\begin{array}{l}
\frac{\partial V_{s}(z, t)}{\partial z}+L_{s} \frac{\partial I_{s}(z, t)}{\partial t}+R_{s} I_{s}(z, t)=V_{f}(z, t) \\
\frac{\partial I_{s}(z, t)}{\partial z}+C_{s} \frac{\partial V_{s}(z, t)}{\partial t}+G_{s} V_{s}(z, t)=I_{f}(z, t)
\end{array}\right.
$$

Inner System

$$
\left\{\begin{array}{l}
\frac{\partial V_{w}(z, t)}{\partial z}+L_{w} \frac{\partial I_{w}(z, t)}{\partial t}+R_{w} I_{w}(z, t)=Z_{t} I_{s}(z, t) \\
\frac{\partial I_{w}(z, t)}{\partial z}+C_{w} \frac{\partial V_{w}(z, t)}{\partial t}+G_{w} V_{w}(z, t)=0
\end{array}\right.
$$

Where $V_{s}$ is the shield-to-ground voltage, $I_{s}$ is the current flowing between the external shield and the ground, $V_{w}$ is the wire-to-shield voltage and $I_{w}$ is the current of the wire.

$L_{s}, C_{s}, R_{s}$, and $G_{s}$ are the per-unit-lenght (p.u.l) inductance, capacitance, resistance and conductance of the outer system, respectively, while $L_{w}, C_{w}, R_{w}$ and $G_{w}$ are the p.u.l inductance, capacitance, resistance, and conductance matrices of the inner system.

$Z_{t}$ is the transfer impedance, In case of braided shield, the transfer impedance is given by the complex expression [13-14]

$$
Z_{t}=Z_{d}+j \omega L_{t}
$$

Where $Z_{d}$ is the diffusion term, and $L_{t}$ is the inductance which accounts for the field penetrating through the braid apertures. The expression of both $Z_{d}$ and $L_{t}$ in terms of the braid weave parameters can be found in [13-14]. In our application, we used a simplified expression [8] $Z_{t}=R_{t}+j \omega L_{t}$, where $R_{t}$ is the constant p.u.l. transfer resistance of the shield.

$V_{f}(z, t)$ and $I_{f}(z, t)$ are distributed sources that represent external excitation of the transmission line. These source terms can be written solely in terms of the incident electric field using Faraday's law. For coaxial shielded cable, shown in Fig. 2, we have: 


$$
\begin{gathered}
V_{f}(z, t)=\left[E_{z}^{i n c}(h, z, t)-E_{z}^{i n c}(0, z, t)\right]-\frac{\partial}{\partial z} \int_{0}^{h} E_{x}^{i n c}(x, z, t) d x \\
I_{f}(z, t)=-C_{s} \frac{\partial}{\partial t} \int_{0}^{h} E_{x}^{i n c}(x, z, t) d x
\end{gathered}
$$

where $h$ is the height of the line, and $E_{z}^{i n c}(h, z, t)$ and $E_{x}^{i n c}(h, z, t)$ are the horizontal and vertical components of the incident electric field, respectively.

The incident field, in the absence of the line, can be written in the following frequency form

$$
\vec{E}^{i n c}(x, y, z, \omega)=E_{0}\left(e_{x} \overrightarrow{a_{x}}+e_{y} \overrightarrow{a_{y}}+e_{z} \overrightarrow{a_{z}}\right) e^{-j \beta_{x} x} e^{-j \beta_{y} y} e^{-j \beta_{z} z}
$$

Where $e_{x}, e_{y}$ and $e_{z}$ are the components of the incident electric field vector along the $x, y$, and $z$ axes, and are given by:

$$
\left\{\begin{array}{l}
e_{x}=\sin \theta_{E} \sin \theta_{P} \\
e_{y}=-\sin \theta_{E} \cos \theta_{P} \cos \phi_{P}-\cos \theta_{E} \sin \phi_{p} \\
e_{z}=-\sin \theta_{E} \cos \theta_{P} \sin \phi_{P}+\cos \theta_{E} \cos \phi_{P} \\
e_{x}+e_{y}+e_{z}=1
\end{array}\right.
$$

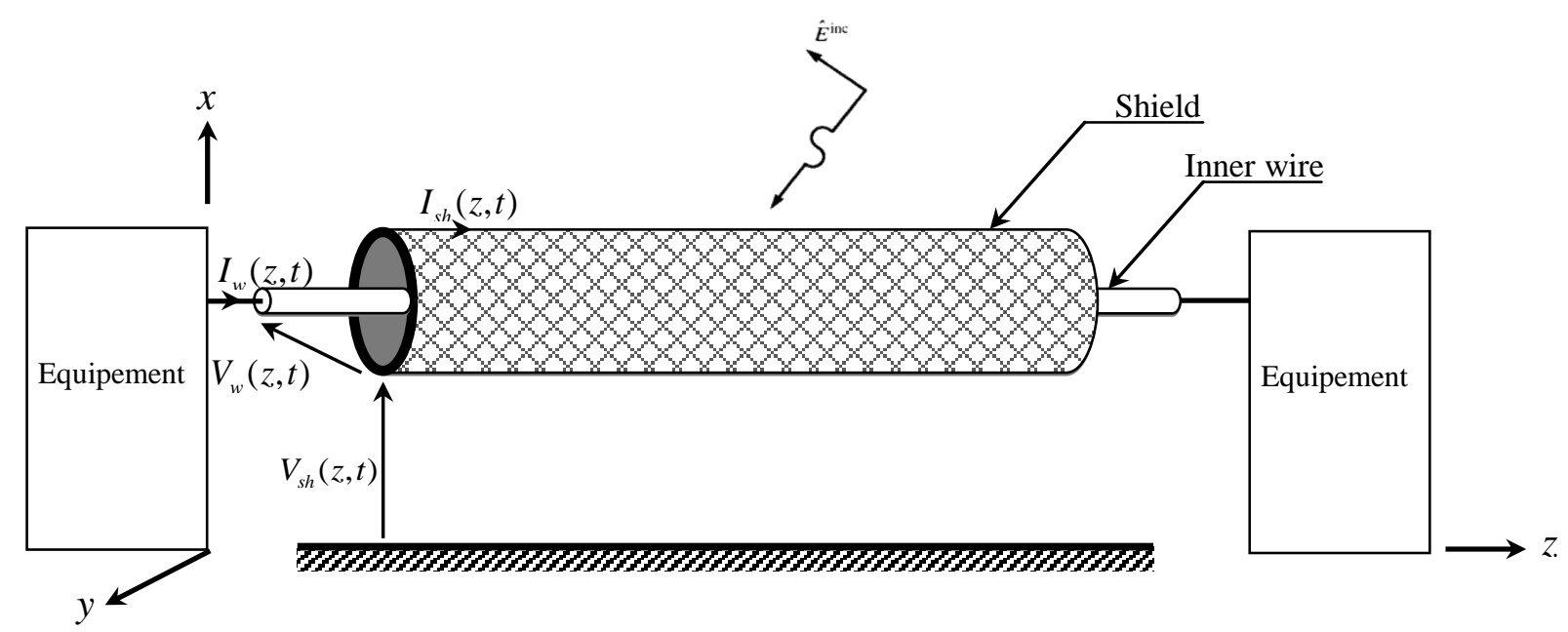

Fig. 1. A Shielded coaxial cable over an infinite and perfectly conducting ground
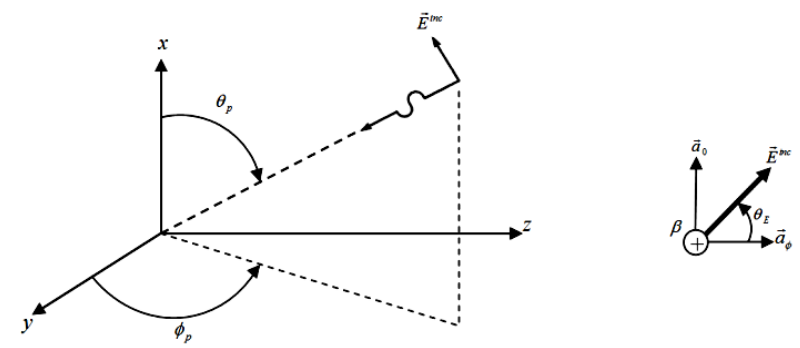

Fig. 2. Definitions of the parameters characterizing the incident field as a uniform plane wave

The angle $\theta_{E}$ characterizes the polarization sort. The polarization is horizontal if $\theta_{E}$ is equivalent to 
zero and vertical if it is equivalent to $90^{\circ}$. The angle $\theta_{p}$ decides the rise with respect to the ground. This one is generally called the incident angle. The angle $\phi_{p}$ gives the propagation direction relative to the axis $O z$.

The components of the phase constant along those coordinate axes are:

$$
\left\{\begin{array}{l}
\beta_{x}=-\beta \cos \theta_{P} \\
\beta_{y}=-\beta \sin \theta_{P} \cos \phi_{P} \\
\beta_{z}=-\beta \sin \theta_{P} \sin \phi_{P}
\end{array}\right.
$$

The phase constant is related to the frequency and properties of the medium as:

$$
\beta=\omega \sqrt{\mu \varepsilon}=\frac{\omega}{v_{0}} \sqrt{\mu_{r} \varepsilon_{r}}
$$

Where $v_{0}=1 / \sqrt{\mu_{0} \varepsilon_{0}}$ is the phase velocity in the space and the medium is characterized by the permeability $\mu=\mu_{0} \mu_{r}$ and permittivity $\varepsilon=\varepsilon_{0} \varepsilon_{r}$.

\section{B. Equivalent Circuit Model for conducted Immunity: Outer system}

In order To solve the equations (1) and (2) we use the 'discrete line' model. For this reason, the cable is discretized in the form of cell; the length of each cell is $\Delta z=\lambda / 10$.

Using Branin's method, each cell can be written in the case of conducted mode as [12]:

$$
\left\{\begin{array}{l}
V_{s}\left(z_{0}, t\right)=Z_{c s} I_{s}\left(z_{0}, t\right)+V_{b r}^{\prime} \\
V_{s}\left(z_{0}+\Delta z, t\right)=-Z_{c s} I_{s}\left(z_{0}+\Delta z, t\right)+V_{b i}^{\prime}
\end{array}\right.
$$

In (10), $Z_{c s}$ represents the characteristic impedance of the outer system. Using the first term of the Taylor series expansion, we obtain [12]

Where

$$
Z_{c s}=\sqrt{\frac{R_{s}+j L_{s} \omega}{j C_{s} \omega}} \approx R_{c s}+\frac{1}{j C_{s f} \omega} \quad R_{s} \ll L_{s} \omega
$$

$$
R_{c s}=\sqrt{\frac{L_{s}}{C_{s}}} \quad \text { and } \quad C_{s f}=\frac{2 L_{s}}{R_{s} R_{c s}}
$$

Eq. (10) becomes

$$
\left\{\begin{array}{l}
V_{s}\left(z_{0}, t\right)=R_{c s} I_{s}\left(z_{0}, t\right)-\frac{R_{c s} R_{s}}{2 \omega^{2} L_{s}} I_{s}^{\prime}\left(z_{0}, t\right)+V_{b r}^{\prime} \\
V_{s}\left(z_{0}+\Delta z, t\right)=-R_{c s} I_{s}\left(z_{0}+\Delta z, t\right)+\frac{R_{c s} R_{s}}{2 \omega^{2} L_{s}} I_{s}^{\prime}\left(z_{0}+\Delta z, t\right)+V_{b i}^{\prime}
\end{array}\right.
$$

Where 


$$
\left\{\begin{array}{l}
V_{b r}^{\prime}=e^{-\alpha_{s} \Delta z}\left[V_{s}\left(z_{0}+\Delta z, t-T_{s}\right)-R_{c s} I_{s}\left(z_{0}+\Delta z, t-T_{s}\right)+\frac{R_{c s} R_{s}}{2 \omega^{2} L_{s}} I_{s}^{\prime}\left(z_{0}+\Delta z, t-T_{s}\right)\right] \\
V_{b i}^{\prime}=e^{-\alpha_{s} \Delta z}\left[V_{s}\left(z_{0}, t-T_{s}\right)+R_{c s} I_{s}\left(z_{0}, t-T_{s}\right)-\frac{R_{c s} R_{s}}{2 \omega^{2} L_{s}} I_{s}^{\prime}\left(z_{0}, t-T_{s}\right)\right]
\end{array}\right.
$$

The characteristic impedance in this case, is presented as a characteristic resistance $R_{c}$ and capacit $C_{f}$ as shown in Fig. 3. Where $T_{s}$ is the one-way delay of the shield, and is denoted by $T_{s}=\Delta z \sqrt{L_{s} C_{s}}$ With the same estimation, the constant of propagation gets to be:

$$
\gamma_{s}=\alpha_{s}+j \beta_{s}=\frac{R_{s}}{2 R_{c}}+j \omega \sqrt{L_{s} C_{s}}
$$

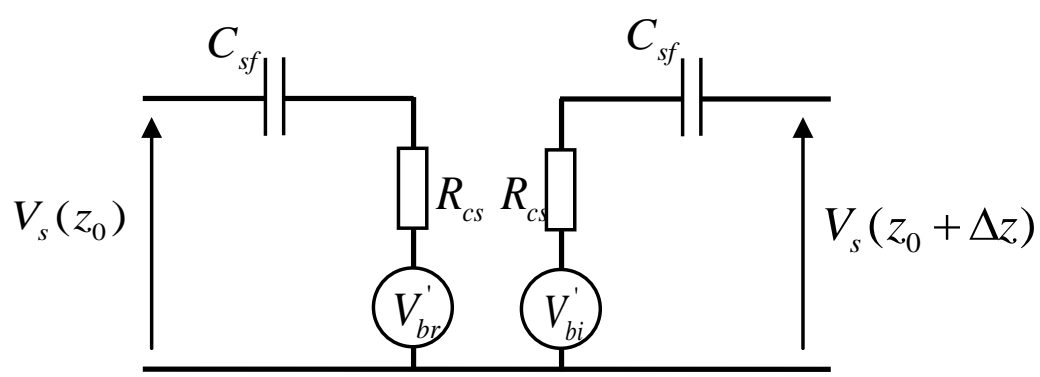

Fig. 3. Circuit model of each cell of the outer system: shield

C. Equivalent circuit model for conducted immunity: Inner system

Using the same procedure, the inner system can be written for each cell as

$$
\begin{aligned}
& V\left(z_{0}, t\right)=R_{c} I_{w}\left(z_{0}, t\right)-\frac{R_{c} R_{w}}{2 \omega^{2} L_{w}} I_{w}^{\prime}\left(z_{0}, t\right)+V_{r}^{\prime} \\
& V\left(z_{0}+\Delta z, t\right)=-R_{c} I_{w}\left(z_{0}+\Delta z, t\right)+\frac{R_{c} R_{w}}{2 \omega^{2} L_{w}} I_{w}^{\prime}\left(z_{0}+\Delta z, t\right)+V_{i}^{\prime}
\end{aligned}
$$

Where

$$
\begin{aligned}
& \left\{\begin{array}{l}
V_{i}^{\prime}=e^{-\alpha \Delta z}\left\{V_{w}\left(z_{0}, t-T_{r}\right)+R_{c w} I_{w}\left(z_{0}, t-T_{r}\right)-\frac{R_{c} R_{w}}{2 \omega^{2} L_{w}} I_{w}^{\prime}\left(z_{0}, t-T_{r}\right)-Z_{t} I_{s}\left(z_{0}+\Delta z\right)\right\} \\
V_{r}^{\prime}=e^{-\alpha \Delta z}\left\{V_{w}\left(z_{0}+\Delta z, t, t-T_{r}\right)-R_{c w} I_{w}\left(z_{0}+\Delta z, t-T_{r}\right)+\frac{R_{c} R_{w}}{2 \omega^{2} L_{w}} I_{w}^{\prime}\left(z_{0}+\Delta z, t-T_{r}\right)\right\}+Z_{t} I_{s}\left(z_{0}+\Delta z\right)
\end{array}\right. \\
& T_{r}=\Delta z \sqrt{L_{w} C_{w}}
\end{aligned}
$$

Where

$$
R_{c w}=\sqrt{\frac{L_{w}}{C_{w}}} \quad \text { (19) } \quad \text { and } \quad C_{f w}=\frac{2 L_{w}}{R_{w} R_{c w}}
$$

These relations represented as shown in Fig. 4. 


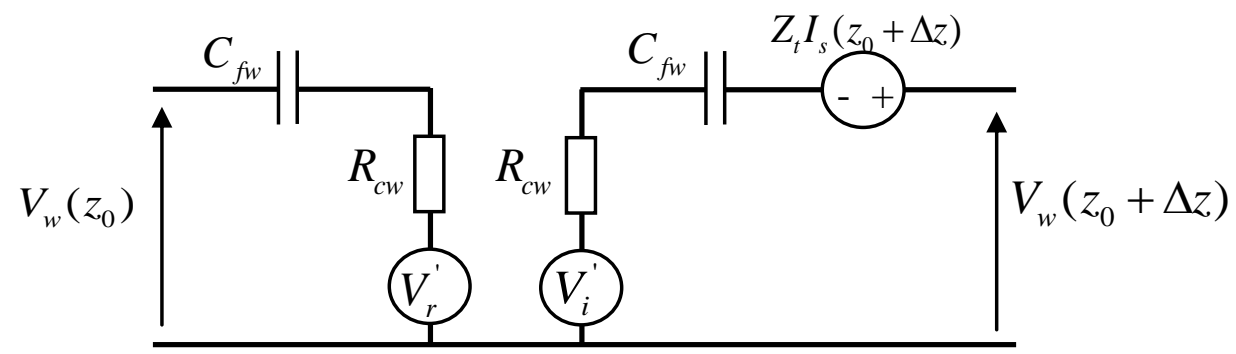

Fig. 4. Circuit model of each cell of the inner system: wire

\section{Equivalent Circuit Model for Radiated Immunity of coaxial shielded cable}

This is the same representation as the conducted immunity by adding generators 'forced' of voltage $\mathrm{E}_{\mathrm{z} 0}$ and $\mathrm{E}_{\mathrm{z} 0+\Delta \mathrm{z}}$, which are representing the coupling between the shield and the incident wave, as shown in Fig. 5.

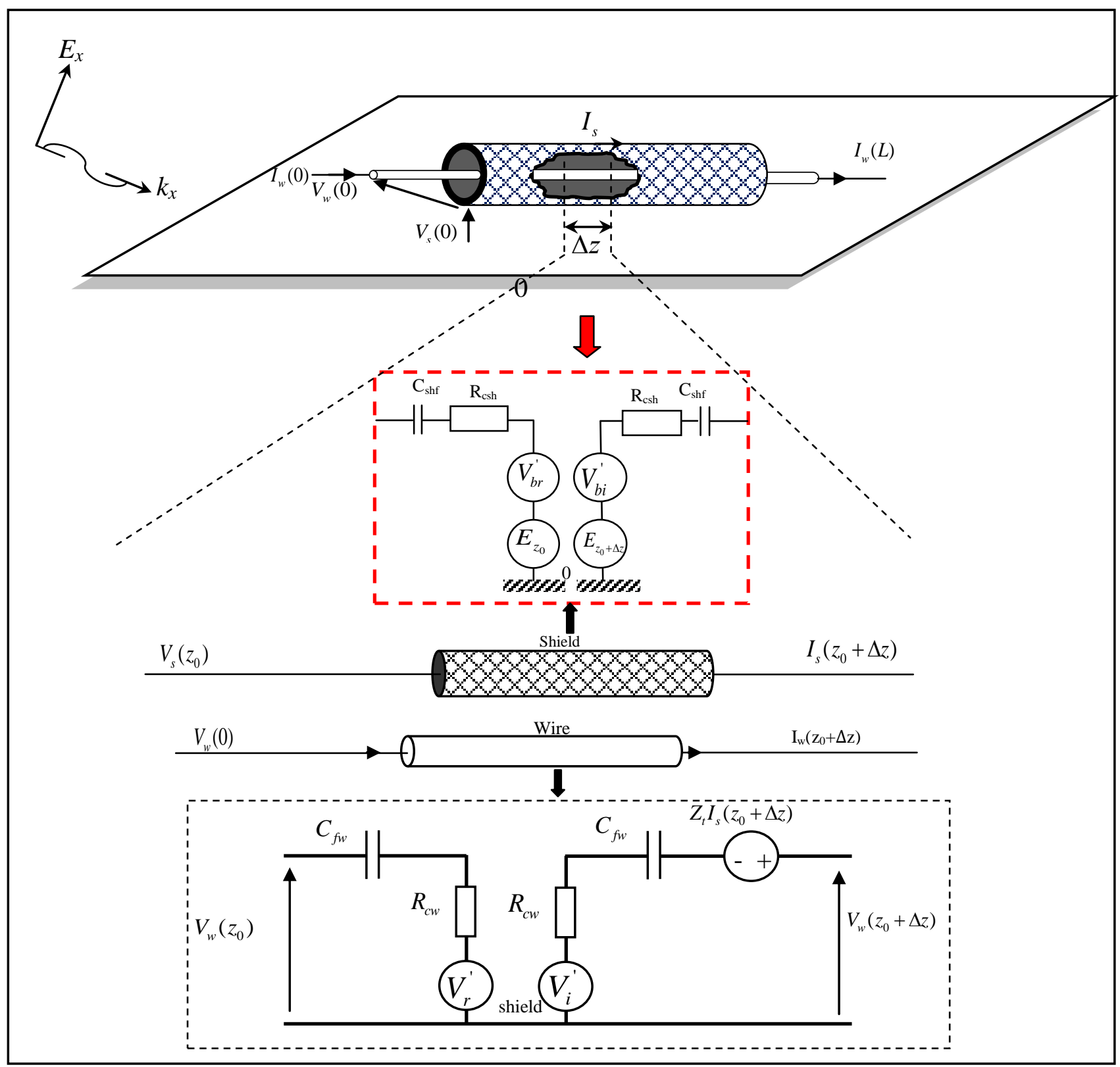

Fig. 5. Equivalent Circuit Model for Radiated Immunity of coaxial shielded cable 
$E_{z 0}$ and $E_{z 0+\Delta z}$ modeling the influence of the incident field in the time domain. For a perfect ground plane, their expressions are defined by [7]

$$
\left\{\begin{array}{l}
E_{z 0}=\alpha_{z 0}\left[\frac{\varepsilon_{0}(t)-\varepsilon_{0}\left(t-T_{z}-T_{z}\right)}{\left(T_{z}+T_{z}\right)}\right] \\
E_{z 0+\Delta z}=\alpha_{z 0+\Delta z}\left[\frac{\varepsilon_{0}\left(t-T_{s}\right)-\varepsilon_{0}\left(t-T_{z}\right)}{\left(T_{s}+T_{z}\right)}\right]
\end{array}\right.
$$

Where $\varepsilon 0(t)$ represents the amplitude of the electric field in the time domain, $\alpha_{z 0}$ et $\alpha_{z 0+\Delta z}$ are the coefficients dependent on the parameters of the line defined by:

$$
\left\{\begin{array}{l}
\alpha_{z 0}=e_{z} T_{x y k} z_{0}-\left(e_{x} x_{k}+e_{y} y_{k}\right)\left(T_{s}+T_{z}\right) \\
\alpha_{z 0+\Delta z}=e_{z} T_{x y k} z_{0}+\left(e_{x} x_{k}+e_{y} y_{k}\right)\left(T_{s}-T_{z}\right)
\end{array}\right.
$$

with $\quad T_{x y k}=\frac{x_{k}}{v_{x}}+\frac{y_{k}}{v_{y}}$

$T_{z}=\frac{\Delta z}{v_{z}}$, if a component wave that propagates along the axis $T_{z}=T_{s}$, in the oposite case $T_{z}=0$.

\section{SIMULATION RESULTS AND VALIDATION}

\section{A. Conducted susceptibility and validation}

The configuration used for the conducted susceptibility is shown in Fig. 6. The length L and the height $\mathrm{h}$ of the cable are $1 \mathrm{~m}$ and $1 \mathrm{~cm}$, respectively. The shield and the inner wire radius are $\mathrm{r}_{\mathrm{s}}=2.5 \mathrm{~mm}$ and $r_{w}=0.25 \mathrm{~mm}$, respectively. The relative perttivity is $\varepsilon_{\mathrm{r}}=2.375$. The values of the transfer resistance and inductance are: $R_{T}=100 \mathrm{~m} \Omega / \mathrm{m}$ and $\mathrm{L}_{T}=0.5 \mathrm{nH} / \mathrm{m}$. The terminal loads between the shield and the ground are $\mathrm{R}_{\mathrm{S} 1}=1 \mathrm{G} \Omega$ and $\mathrm{R}_{\mathrm{S} 2}=154.363 \Omega$, while the inner terminations are matched $\mathrm{R}_{\mathrm{w} 2}=\mathrm{R}_{\mathrm{w} 1}=44 \Omega$.

The lumped current source adopted for the transient analysis is a clock wave of unit amplitude characterized by period $T_{c l k}=20 \mathrm{~ns}$, rise and fall time, and duty cycle $\tau / T_{c l k}=0.5$. Fig. 7 shows the wire-to-shield voltage at the cable ends, which agree well with the results from different methods.

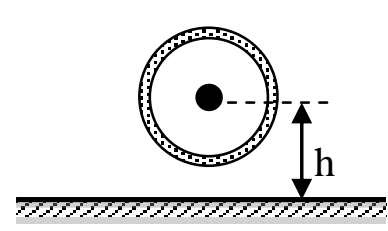

(a)

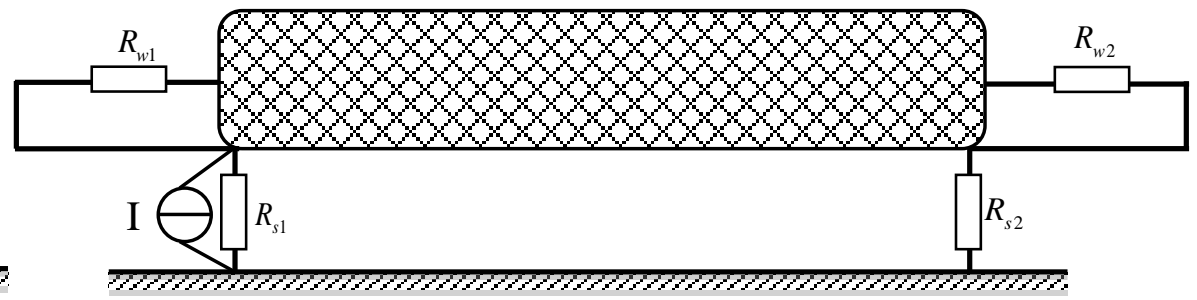

(b)

Fig. 6. (a) Geometrical cross-section of the coaxial cable. (b) Configuration of the simulation for conducted analysis 


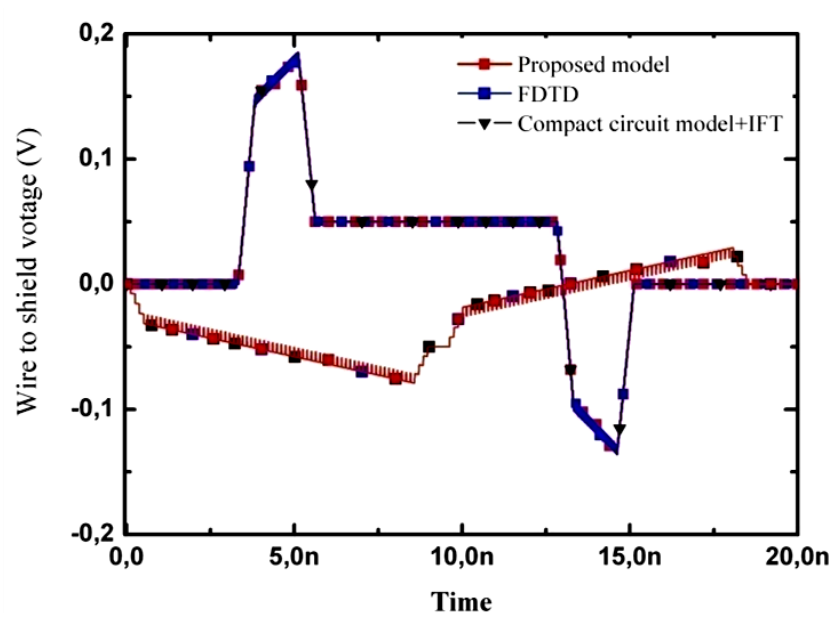

Fig.7. Voltage responses of the inner loads in the transient analysis obtained by different methods

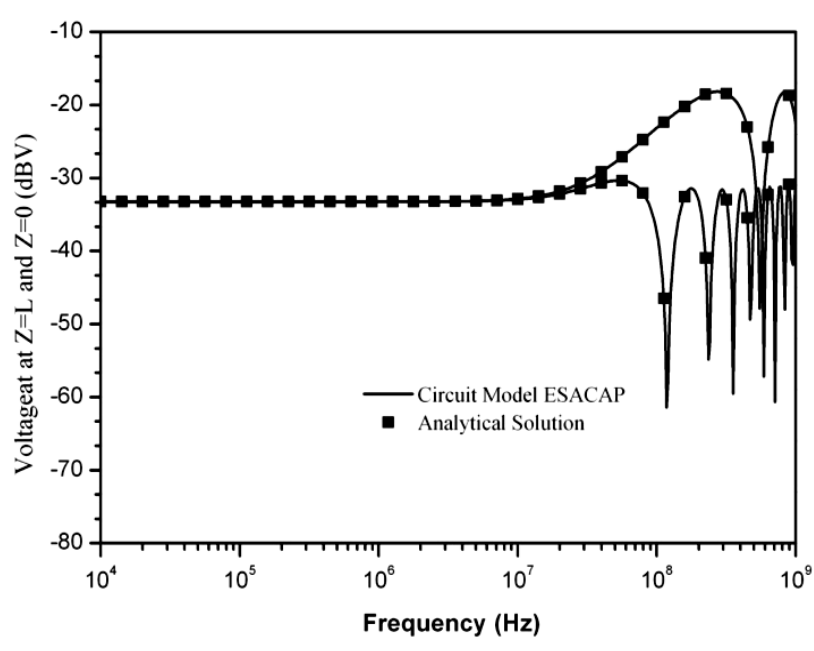

Fig. 8. Magnitude of the frequency responses in decibels of the inner terminations

The wire-to-shield voltage at the cable ends acquired by the proposed model is appeared in Fig.7 together with the outcomes determined by the FDTD [15], where the "FDTD" implies the finite difference time domain solution to the transmission-line equations of the cable, and by the compact circuit model proposed in [10]. They are in very good agreement with each other.

The lumped current source is set to $1 \mathrm{~A}$ for the frequency-domain analysis. Fig. 8 demonstrates the magnitude of the frequency responses of the inner terminators acquired by the proposed model. The outcomes got by the ESACAP test system are in great concurrence with the analytical solution [16].

As shown in Fig. 8. The coupling into end side load is clearly stronger than in near side load, because the injection is asymmetrically located on near side of external shield, the total coupling is under a flat envelop and the anti-resonance frequencies are located as by the following formula at $\mathrm{f}=\mathrm{n} \times\left(3.10^{8} 2 / \lambda \sqrt{\varepsilon}\right), \mathrm{n}=1,3,5 \ldots$

\section{B. Radiated Susceptibility Analysis of Coaxial Cable}

The analysis of the radiated immunity is carried out on the coaxial cable as shown in Fig. 9. The shield radius and the inner wire radius are $0.25 \mathrm{~mm}$ and $0.108 \mathrm{~mm}$, respectively. The cable's characteristic impedance is $Z_{c}=50 \Omega$, and the relative permittivity $\varepsilon_{r}$ of the internal dielectric filling is 1.77 . The value of the transfer impedance is set to $R_{T}=1 \Omega / \mathrm{m}$ and $L_{T}=0 \mathrm{H} / \mathrm{m}$. The height $\mathrm{h}$ and the length $\mathrm{L}$ are $5.25 \mathrm{~mm}$ and $1 \mathrm{~m}$, respectively. The internal conductor is adapted with $\mathrm{Za}=\mathrm{Zb}=50 \Omega$. The incident field is modeled by the double exponential pulse $E(t)=k E_{0}[\exp (-A t)-\exp (-B t)], \quad$ assuming $\quad E_{0}=50 \mathrm{KV} / \mathrm{m}, \quad k=1.3, \quad A=6 \cdot 10^{8} \mathrm{~s}^{-1}$, 
$B=4 \cdot 10^{7} s^{-1}$, is used for the time domain analysis, while the electric field of $1 \mathrm{~V} / \mathrm{m}$ magnitude for the frequency domain analysis.

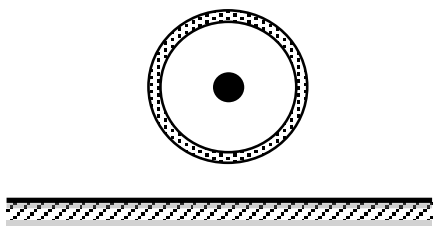

(a)

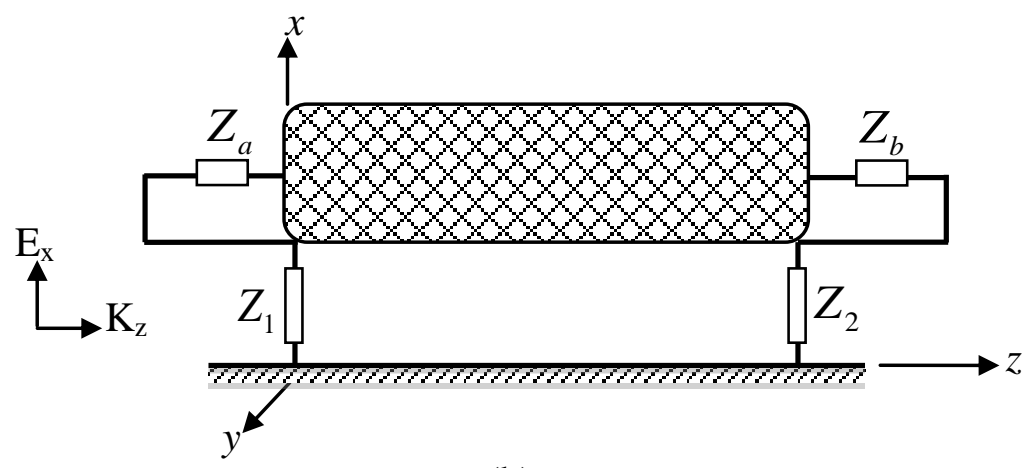

(b)

Fig. 9. (a) (a) Geometrical cross-section of the coaxial cable. (b) Configuration of the simulation for radiated analysis

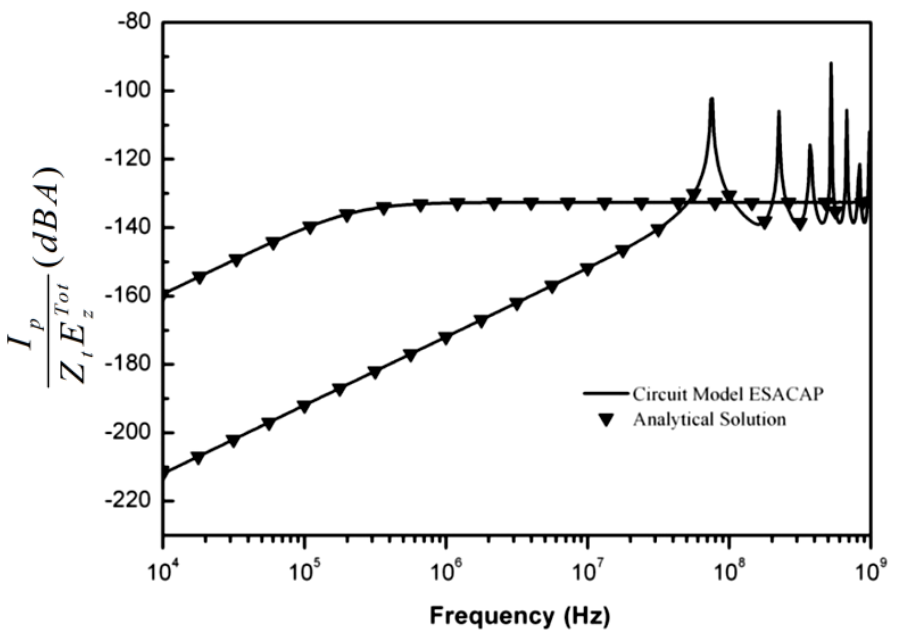

Fig. 10. Currents induced on shielded cable in dBA excited by uniform Ex- Kz, obtained by different methods (normalized to $\left.Z_{t} E_{z}^{T o t}\right)$.

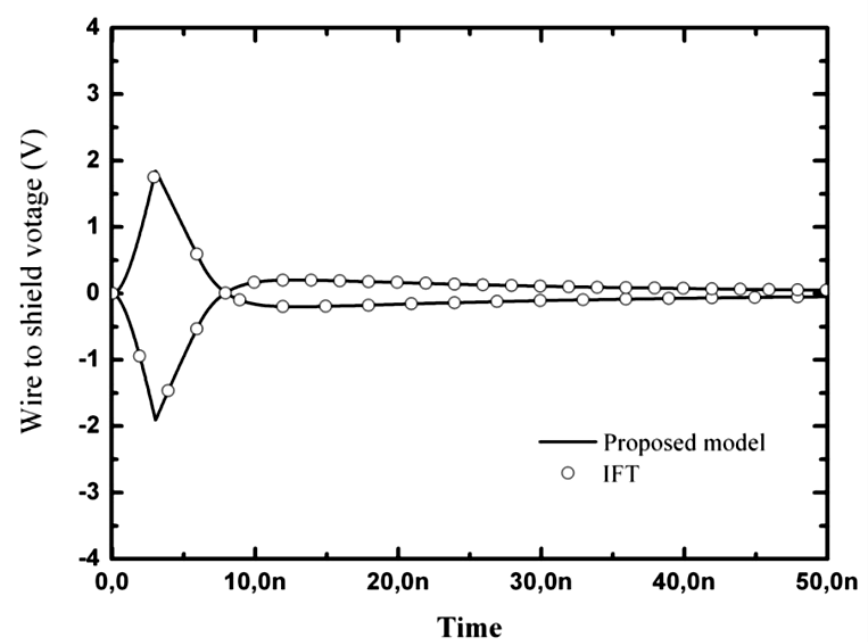

Fig. 11. Voltages induced at the cable ends excited by an incident plane wave Ex- Kz, obtained by different methods 
The shield is short circuited on the right $\left(\mathrm{Z}_{2}=0.5 \Omega\right)$. At the output of the coaxial we recover the current into dBA, which is matched with the canonic results published by Smith [16], as shown in Fig.10.

When the shield is open on the left $\left(Z_{1}=0.5 \Omega\right)$ a towering resonances at about $\lambda / 4$, as shown in Fig.10. To drastically diminish the coupling to internal wire, a two-side grounded configuration for the shield must be utilized. Fig. 11 demonstrates the voltage reactions at the inner loads of the cable in the time-domain analysis acquired by the diverse methods, when $\mathrm{Z}_{1}=\mathrm{Z}_{2}$ equivalent to the characteristic impedance of the shield-to-ground $\left(\mathrm{Z}_{1}=\mathrm{Z}_{2}=244.5 \Omega\right)$. The arrangements of the distinctive techniques concur exceptionally well.

\section{Variation effect of incident plane wave on Coaxial cable with unmatched line loads, over ground plane}

Fig. 12 shows a coaxial cable of $1 \mathrm{~m}$ length at $5.25 \mathrm{~mm}$ above a perfectly conducting ground plane, the shield radius $R_{\mathrm{sh}}$ and the inner wire radius $r_{\mathrm{w}}$ are $0.25 \mathrm{~mm}$ and $0.0716 \mathrm{~mm}$, respectively, with dielectric constant $\varepsilon_{\mathrm{r}}=2.25$. The loads $\mathrm{R}_{1}$ and $\mathrm{R}_{2}$ between the inner wire and the shield at the two terminations are $R_{1}=10 \Omega$ and $R_{2}=1000 \Omega$. The value of the transfer impedance is set to $R_{T}=0.01 \Omega / m$ and $\mathrm{L}_{\mathrm{T}}=1 \mathrm{nH} / \mathrm{m}$. The incident electromagnetic field is a plane wave, while the incident field $\mathrm{E}=1 \mathrm{~V} / \mathrm{m}$.

The Analysis performed for three reference field directions as described in Fig. 7 are as follow:

a) $E_{x}-K_{z}$ : The polarization direction of electric field is along $z$ axis.

b) $E_{x}, K_{y}$ : The polarization direction of electric field is along y axis

c) $E_{z}, K_{x}$ : The polarization direction of electric field is along $x$ axis

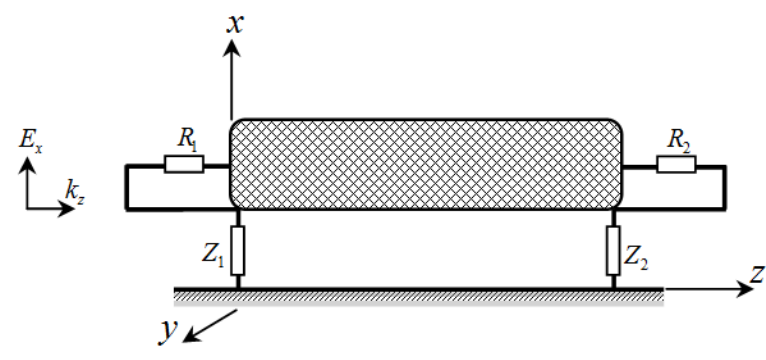

Fig. 12a. Case 1- Coaxial cable over an infinite and perfectly conducting ground excited by an incident plane with $\phi_{p}=-90^{\circ}, \theta_{p}=90^{\circ}$ and $\theta_{E}=90^{\circ}$

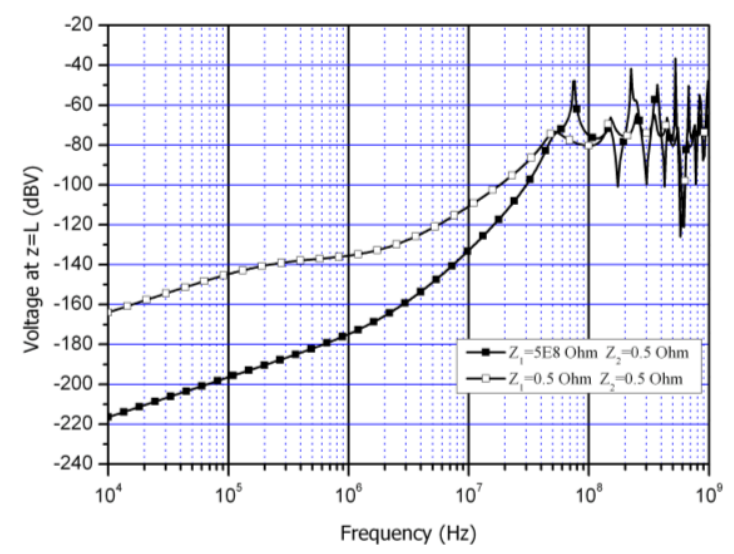

Fig. 13a. Case 1- Voltage responses at the cable ends in the frequency analysis with the incident wave with $\phi_{p}=-90^{\circ}, \theta_{p}=90^{\circ}$ and $\theta_{E}=90^{\circ}$ 


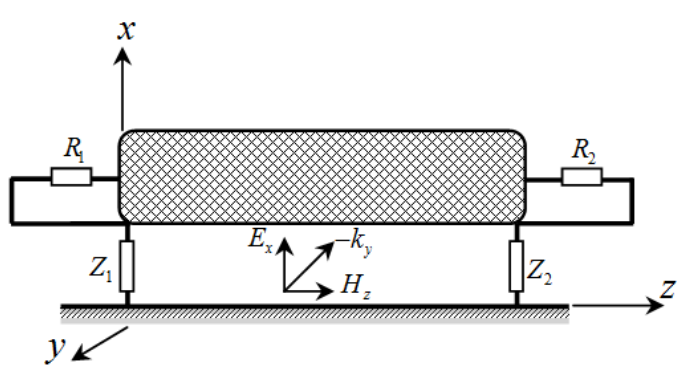

Fig. 12b. Case 2- Coaxial cable over an infinite and perfectly conducting ground excited by an incident plane with $\phi_{p}=0^{\circ}, \theta_{p}=90^{\circ}$ and $\theta_{E}=90^{\circ}$

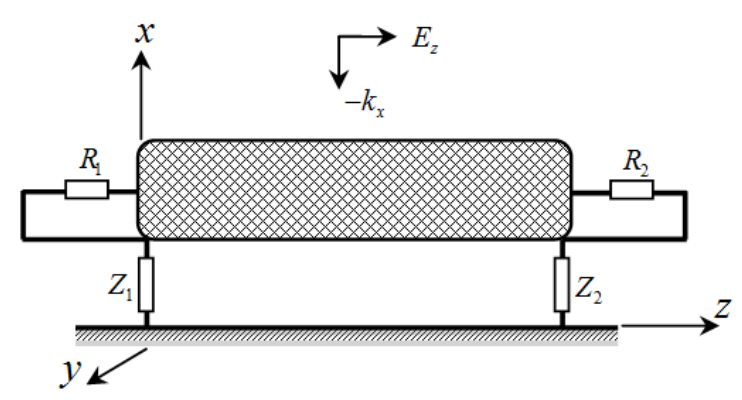

Fig. 12c. Case 3- Coaxial cable over an infinite and perfectly conducting ground excited by an incident plane with $\phi_{p}=0^{\circ}, \theta_{p}=0^{\circ}$ and $\theta_{E}=0^{\circ}$

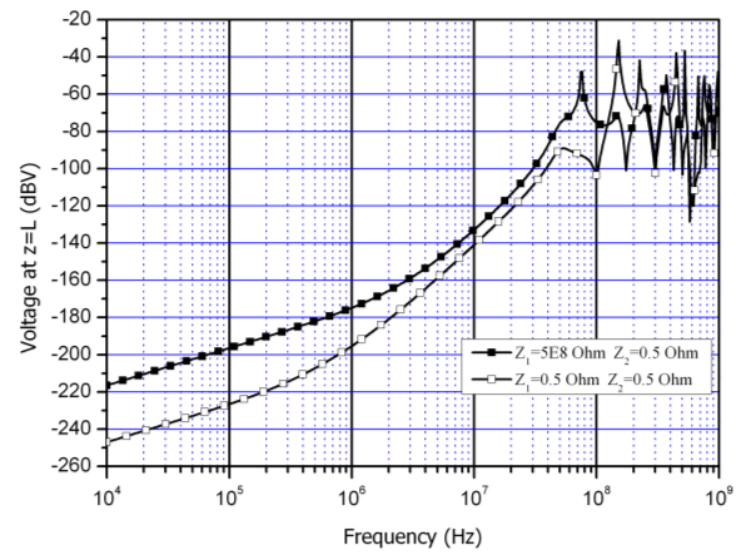

Fig. 13b. Case 2- Voltage responses at the cable ends in the frequency analysis with the incident wave with $\phi_{p}=0^{\circ}, \theta_{p}=90^{\circ}$ and $\theta_{E}=90^{\circ}$

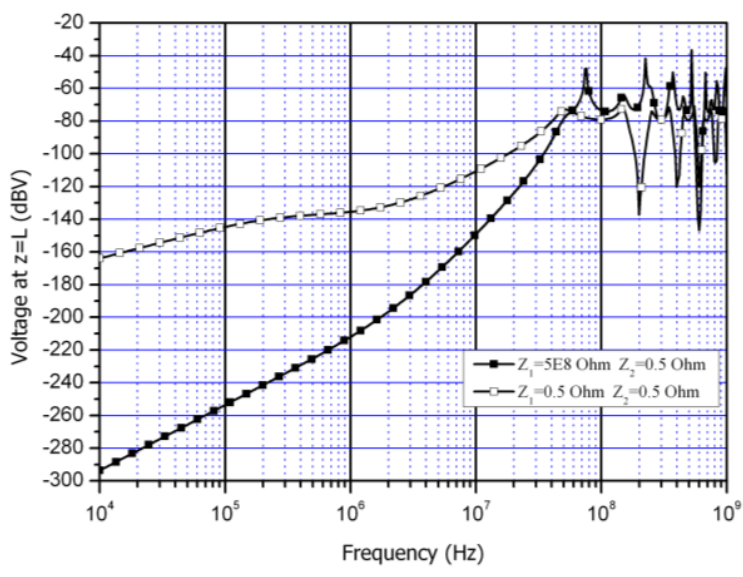

Fig. 13c. Case 3- Voltage responses at the cable ends in the frequency analysis with the incident wave with $\phi_{p}=0^{\circ}, \theta_{p}=0^{\circ}$ and $\theta_{E}=0^{\circ}$

The two different curves for each case, with similar field illuminations, correspond to typical applications where the shield is connected to the ground on both sides, or only on one side, mainly at the receiving end. The internal wire is always loaded, as typical for real signal bus, with low resistance on the transmitting side and high resistance on the receiving side.

In Figs. 13, we compare the voltages at the far-end for three reference field directions. For all cases, it is seen that the line resonates at $\lambda /(3 \times 4) \rightarrow f=225 \mathrm{MHz}$ when the shield load is open at the near-end. The value of the open circuit resistance chosen here is $500 \mathrm{M} \Omega$. Also the high impedance resistance values located at opposite ends, for internal line and external shield, provides some phase compensation, practically eliminating the resonance $\lambda / 4 . \rightarrow f=75 \mathrm{MHz}$. However, with short circuit at the ends, it is seen that for cases 1 and 2, eliminates practically all resonance, and the internal immunity is improved more than 20dB. For the case 3, the side vertical illumination excites the shield with maximum efficiency. This is the reason why the line resonates at $\lambda / 2 \rightarrow f=150 \mathrm{MHz}$ and $\lambda /(3 \times 2) \rightarrow f=450 \mathrm{MHz}$, even under short circuit conditions. 


\section{CONCLUSION}

Circuit models were used to analyze the radiated and conducted susceptibilities for lossy shielded coaxial cables. It requires the subdivision of cables into several uniform sections first. Then the voltage and current distributions are obtained using Branin's model. The principle point of interest of these models comprises in the likelihood of utilizing them directly in time and frequency domain analysis. Finally, the introduced approach is verified by comparing its results with other methods.

The proposed model can be also extended to the MTLs in complex systems. This question will be address in future work

\section{REFERENCES}

[1] Bowick C, Blyler J, and Ajluni C.J: RF circuit design, 2nd ed. Amsterdam ; Boston, 2008.

[2] Dhaene T, Martens L, and De Zutter D. Transient simulation of arbitrary nonuniform interconnection structures characterized by scattering parameters, IEEE Trans. Circuits Syst. Fundam. Theory Appl. 1992, vol. 39, no. 11, pp. 928-937.

[3] Burkhart S. C, Wilcox R. B. Arbitrary pulse shape synthesis via nonuniform transmission lines, IEEE Trans. Microw. Theory Tech. 1990, vol. 38, no. 10, pp. 1514-1518.

[4] Khalaj-Amirhosseini M. Analysis of coupled or single nonuniform transmission lines using stepby-step numerical integration, Prog. Electromagn. Res., 2006, vol. 58, pp. 187-198.

[5] Roberts P.P, and Town G.E. Design of microwave filters by inverse scattering," IEEE Trans. Microw. Theory Tech., 1995, vol. 43, no. 4, pp. 739-743.

[6] Lin D.B, Wu F.N, Liu W.S, Wang C.K, and Shih H,Y. Crosstalk and discontinuities reduction on multi-module memory bus by particle swarm optimization, Prog. Electromagn. Res., 2011, vol. 121, pp. 53-74.

[7] Mejdoub Y, Rouijaa H, andGhammaz A. Variation effect of plane-wave incidence on multiconductor transmission lines, Int. J. Microw. Wirel. Technol., 2015, pp. 1-8.

[8] Xie H, Wang J, Fan R, and Liu Y. SPICE Models to Analyze Radiated and Conducted Susceptibilities of Shielded Coaxial Cables, IEEE Trans. Electromagn. Compat., 2010, vol. 52, no. 1, pp. 215-222.

[9] Caniggia S, and Maradei F. Equivalent circuit models for the analysis of coaxial cables immunity, 2003, vol. 2, pp. 881-886.

[10] Caniggia S, and Maradei F. SPICE-Like Models for the Analysis of the Conducted and Radiated Immunity of Shielded Cables, IEEE Trans. Electromagn. Compat., 2004, vol. 46, no. 4, pp. 606616.

[11] Xie H, Wang J, Fan R, and Liu Y. Spice models for radiated and conducted susceptibility analyses of multiconductor shielded cables, Prog. Electromagn. Res., 2010, vol. 103, pp. 241257.

[12] Saih M, Rouijaa H, and Ghammaz A. Circuit models of multiconductor shielded cables: incident plane wave effect," Int. J. Numer. Model. Electron. Netw. Devices Fields, 2016, vol. 29, no. 2, pp. 243-254.

[13] Tesche, F., Ianoz, M., Karlsson, T.: EMC Analysis Methods and Computational Models. New York, Wiley, 1997.

[14] Saih, M., Rouijaa, H., Ghammaz, A.: Crosstalk reduction by adaptation of shielded cables, international Conference on Intelligent Information and Network Technology, Settat Morocco, (2013).

[15] Roden, J.A., Paul, C.R., Gedney, W.T.: Finite-difference, time domain analysis of lossy transmission lines, IEEE Trans. Electromagn. Compat., vol. 38, 1996, pp 15-24.

[16] Albert A. Smith, Jr.: Coupling of external electromagnetic fields to transmission lines, (John Wiley \& Sons, 2nd Edition, 1992). 